\title{
EI Sistema de Telemedicina Militar en España: una aproximación histórica
}

\author{
Hillán García L. ${ }^{1}$, Setién Dodero F. ${ }^{2}$, Del Real Colomo A. ${ }^{3}$ \\ Sanid. mil. 2014; 70 (2): 121-131; ISSN: 1887-8571
}

\begin{abstract}
RESUMEN
La telemedicina es una moderna disciplina con apenas medio siglo de vida cuya evolución e impacto está siendo verdaderamente relevante. En España, los orígenes de la telemedicina hay que buscarlos en las primeras consultas por radio de asistencia sanitaria en alta mar a finales de los años 20. Pero pasarían varias décadas hasta que en 1996 se inaugurara el primer servicio de telemedicina en España cuando se establece una sesión de videoconferencia vía satélite Inmarsat desde la Unidad de Telemedicina del Hospital Militar "Gómez Ulla" en Madrid (España) al centro médico del destacamento desplegado en Mostar (Bosnia-Herzegovina). Se inicia, desde entonces, una importante proyección de la telemedicina en España, y muy especialmente en la Sanidad Militar, al ponerse las bases del que es hoy el Sistema de Telemedicina del Ministerio de Defensa. Este pionero Sistema de Telemedicina ha sabido mantenerse siempre a la vanguardia de los nuevos avances en telemedicina, constituyéndose como un referente internacional en este campo. Es por eso que, en este artículo, nos acercamos a la historia de la telemedicina militar en España, poniendo la tilde en su Sistema de Telemedicina, para conocer mejor el papel desempeñado de la telemedicina en la Sanidad Militar, su evolución, sus limitaciones y fortalezas, así como sus nuevos retos y perspectivas de futuro.
\end{abstract}

PALABRAS CLAVE: Sanidad militar, Telemedicina / historia, Telemedicina / tendencia, Telemedicina / organización y administración, España.

The Military Telemedicine System in Spain: a historical approach

SUMMARY: Telemedicine is a modern discipline with just half a century whose evolution and impact is being truly relevant. In Spain, the origins of telemedicine must be sought in the first radio-medical consultations provided for ships at sea in the late 20s. But, it would pass several decades until 1996 when the first telemedicine service in Spain opened establishing a video conferencing session via Inmarsat satellite from Telemedicine Unit Military Hospital "Gómez Ulla" in Madrid (Spain) to the medical detachment's service deployed to Mostar (Bosnia-Herzegovina). It starts, since then, an important projection of telemedicine in Spain, and especially in the Military Health System, to build the background of, what is today, the Spanish Ministry of Defence's Telemedicine System. This pioneering Telemedicine System has kept at the forefront of new developments in telemedicine, becoming an international leader in this field. That's why, in this article, we approach the history of military telemedicine in Spain, putting the accent in its Telemedicine System, to better understand the role of telemedicine in the Military Health System, its evolution, its limitations and strengths, as well as its new challenges and future prospects.

KEY WORDS: Military medicine, Telemedicine/history, Telemedicine/trends, Telemedicine/organization \& administration, Spain

\section{INTRODUCCIÓN}

Las tecnologías de la información y las comunicaciones (TIC) han revolucionado el mundo en que vivimos. Con la era digital se inicia un vertiginoso viaje hacia nuevas formas de comunicación, de trabajo, producción y generación de conocimiento que no para de crecer exponencialmente. Las TIC evolucionan a un ritmo tal que supone un reto continuo el saber aprovechar todas sus posibilidades.

En el área de la medicina las ventajas del uso de las TIC han sido y están siendo verdaderamente relevantes, siendo en el campo de la telemedicina donde adquieren mayor protagonismo. La

${ }^{1}$ Lic. Documentalista.

${ }^{2}$ Ing. Telecomunicaciones.

${ }^{3}$ Cte. Médico.

Hospital Central de la Defensa Gómez Ulla. Servicio de Telemedicina. Madrid. España.

Dirección para correspondencia: Antonio del Real Colomo. Servicio de Telemedicina. Hospital Central de la Defensa "Gómez Ulla". Glorieta del Ejército s/n. 28047 Madrid. España. aereacol@et.mde.es

Recibido: 17 de mayo de 2013

Aceptado: 20 de junio de 2013 telemedicina, entendida en su concepto más amplio como la medicina practicada a distancia, rompe las barreras tiempo-espacio para poder ofrecer asistencia sanitaria cuándo y dónde se necesite. Además, con los avances de las TIC, la optimización y mejora continua es cada vez más significativa, abriéndose atractivos horizontes y grandes expectativas.

En la actualidad, la telemedicina está en plena expansión; en un contexto de dificultades económicas, la utilización eficiente de los recursos cobra especial relevancia y la telemedicina recibe las miradas de la comunidad científica, y de los policy makers como medio sostenible y de calidad para garantizar el estado del bienestar de una población con una esperanza de vida larga (se prevé que para el 2030, el 22\% de la población de los países ricos llegue a tener 65 años o más ${ }^{1}$ ) y aquejada de enfermedades crónicas; las Naciones Unidas en su última cumbre sobre la salud, advertía de los peligros sobre el creciente número de enfermedades crónicas en todo el mundo ${ }^{1}$. Por otra parte, la necesidad de aumentar el alcance de la atención médica a lugares remotos y de difícil acceso en países pobres y emergentes hace prever un crecimiento acelerado de la telemedicina, sobre todo en determi- 


\section{Hillán García, et al.}

nadas regiones. Según un reciente informe de la consultora $B C C$ Research el mercado mundial de la telemedicina fue valorado en 11,6 billones de dólares en 2011 y se prevé que siga creciendo hasta alcanzar los 2,3 billones en 2016, lo que representa una tasa de crecimiento anual compuesta del 18,6 \% ${ }^{2}$.

En el caso de España, y a pesar de una introducción tímida de la telemedicina en el sistema sanitario español, se ha percibido una rápida evolución y buena acogida de los nuevos avances en esta disciplina, de tal forma que cada vez es más habitual la prestación de servicios de telemedicina en la Sanidad Pública. Sus inicios hay que buscarlos dentro de la Fuerzas Armadas españolas (FAS); pioneros en el uso ${ }^{3}$ y desarrollo de esta disciplina, se han mantenido siempre a la vanguardia de los nuevos avances en telemedicina, constituyéndose como un referente clave de buenas prácticas en este campo. Asimismo, tanto las necesidades intrínsecas de su actividad, como el privilegio de gozar de recursos tecnológicos avanzados, han hecho que las Fuerzas Armadas hayan sabido aprovechar las ventajas de la telemedicina en el día a día de su sanidad.

Es por este motivo que nos queremos acercar a la historia de la telemedicina militar en España; la evolución que ha experimentado, las condiciones y recursos con los que se han ido contando, el papel desempeñado a lo largo del tiempo, así como los nuevos retos y perspectivas de futuro.

\section{PRIMEROS PASOS EN TELEMEDICINA}

El inicio de la telemedicina actual se imagina en abril de 1924 cuando la revista americana Radio News publica en portada la posible llegada del Doctor por radio e ilustra cómo podría funcionar este sistema mediante una pantalla de televisión y un altavoz $z^{4}$.

Pero pasaría más de un cuarto de siglo, hasta que a finales de los años 50, se instalaron los primeros sistemas de circuito cerrado de televisión bidireccional por microondas para la tele-consulta y la tele-educación. La introducción de la televisión con fines médicos se considera el primer gran impulso para la telemedicina, que posibilitó, en 1964, el primer enlace de video interactivo entre el instituto de psiquiatría de Nebraska en Omaha y el hospital estatal Norfolk separados por 112 millas ${ }^{5}$. Y un poco más tarde, en el año 1967, se instaló el primer sistema completo de telemedicina entre el centro de atención primaria del Boston's Logan Airport y el Massachusetts General Hospital ${ }^{5}$ para la comunicación médico-paciente en tiempo real. Los primeros estudios demostrarían la viabilidad y eficacia en la transmisión de historiales médicos, radiografías, y datos de laboratorio, así como el diagnóstico de patologías desde un área remota a través de la televisión.

Con la aparición de los primeros satélites en 1957 y las grandes expediciones espaciales de los años 60, comienza una carrera por alcanzar nuevas formas de monitorización y asistencia médica a distancia, capaces de mantener a los astronautas varios días en el espacio expuestos a una gravedad cero. Las preocupaciones de los científicos de la NASA por controlar las facultades físicas y psicológicas de los astronautas que debían enfrentarse a condiciones extremas, posibilitó el desarrollo de sofisticados sistemas de telemetría biomédica cuya función era recibir de forma continua datos sobre el ritmo cardiaco, la presión sanguínea sistólica y diastólica, y la temperatura de los astronautas ${ }^{6}$. A medida que las exigencias de vuelo eran mayores, fueron perfeccionándose

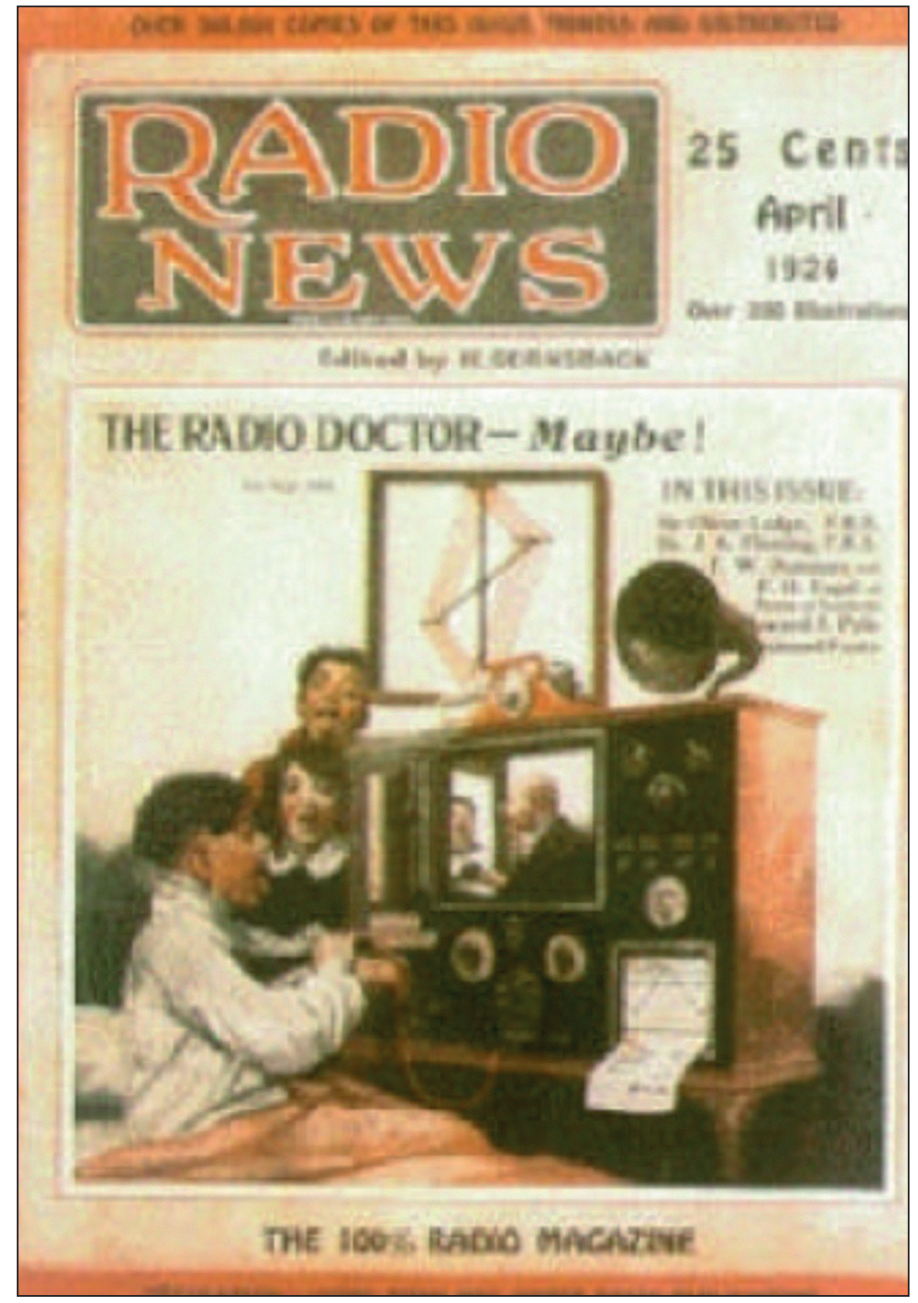

Figura 1. Portada de la Revista "Radio News”, 1924.

estos sistemas hasta alcanzar grandes posibilidades de monitorización, diagnóstico y tratamiento en vuelo.

Estos avances en telemetría biomédica impulsarían que se invirtiesen más recursos en $\mathrm{I}+\mathrm{D}$ en este área. Por ese motivo, durante los años 70, agencias gubernamentales de EEUU financiaron varios proyectos de investigación sobre asistencia sanitaria a distancia, con el propósito de analizar la viabilidad y fiabilidad del uso de programas interactivos de telecomunicaciones para el diagnóstico y tratamiento de enfermedades en áreas remotas o de difícil acceso.

Muchos de estos proyectos se desarrollaron en áreas rurales donde existían mayores carencias de servicios sanitarios. Como ejemplo pionero de este tipo de proyectos, suele citarse el STARPAHC (the Space Technology Applied to Rural Papago Advanced Health Care) que proporcionaba asistencia sanitaria con el sistema de telemetría de la NASA a los indios de la tribu Papago en su reserva del Estado de Arizona ${ }^{6}$.

A pesar de que muchos de estos proyectos quedaron sin concluir debido a los recortes en su financiación y las propias limitaciones de unos equipos tecnológicos aún rudimentarios, esta primera etapa de la telemedicina evidenciaría el gran valor de las telecomunicaciones en la provisión de servicios sanitarios en áreas remotas o de difícil acceso.

Los años 90 vendrán caracterizados por el desarrollo de equipos electrónicos de telecomunicación y la disminución en 


\section{El Sistema de Telemedicina Militar en España: una aproximación histórica}

los costes de su producción. Es en estos años cuando se introduce la palabra telemedicina como término MESH en Medline ${ }^{5} \mathrm{y}$ surgen nuevas políticas promovidas por organismos internacionales para la sistematización y estandarización del conocimiento adquirido hasta el momento para su aplicación en el campo de la salud. Se inicia, entonces, la llamada segunda etapa de la telemedicina que llega hasta nuestros días.

\section{EL CONCEPTO DE TELEMEDICINA}

El concepto de telemedicina, como demuestra las múltiples definiciones existentes, es un término vivo ${ }^{7}$ que va evolucionando a lo largo del tiempo en función de los avances tecnológicos y las necesidades médicas de la sociedad del momento. No obstante, todas las definiciones giran en torno a una idea clave: la comunicación y el intercambio de información.

Si se acude a su origen etimológico, el término telemedicina proviene de la palabra griega tele que significa a la distancia y de la palabra griega mederi o del latin medecus que significa curar $^{8}$. Por lo que, en su concepción más primaria y literal, se podría decir que la telemedicina es la curación a distancia.

Desde que se acuñara por primera vez el término en 1970 por Thomas Bird, se han sucedido múltiples definiciones hasta que en 1997 la Organización Mundial de la Salud (OMS) adoptara la suya propia:

"Telemedicina es el suministro de servicios de atención sanitaria, en los casos en que la distancia es un factor crítico, llevado a cabo por profesionales sanitarios que utilizan tecnologías de la información y la comunicación para el intercambio de información válida para hacer diagnósticos, prevención y tratamiento de enfermedades, formación continuada de profesionales en atención a la salud así como para actividades de investigación y evaluación, con el fin de mejorar la salud de las personas y de sus comunidades".?

Otras definiciones de referencia son:

Asociación Americana de Telemedicina (ATA): "Telemedicina es el uso de información médica intercambiada entre distintos lugares por medio de comunicaciones electrónicas para mejorar el estado de salud de los pacientes. La telemedicina incluye una creciente variedad de aplicaciones y servicios que usan vídeo bidireccional, correo electrónico, teléfonos inteligentes, herramientas inalámbricas y otras formas de tecnología de las telecomunicaciones" 9.

INSALUD: "Telemedicina es la utilización de las tecnologías de la información y de las comunicaciones como un medio de proveer servicios médicos, independientemente de la localización tanto de los que ofrecen el servicio, los pacientes que lo reciben, y la información necesaria para la actividad asistencial" 10 .

La esencia de la Telemedicina es la provisión de servicios e información a los usuarios en su propio entorno, en lugar de trasladarlos a centros sanitarios. Lo cual exige disponer de nuevas herramientas para superar barreras socioeconómicas, culturales y geográficas en la atención sanitaria.

En última instancia, los objetivos de la telemedicina son:

- Extender el alcance de la asistencia sanitaria a lugares donde no existe, o de difícil acceso.

- Mejorar los procesos de diagnóstico, prescripción y toma de decisiones.

- Proporcionar continuidad asistencial en casos que precisen vigilancia continua.

- Rentabilizar y optimizar recursos materiales y humanos, centralizando el personal en un menor número de centros sanitarios que actúan como centros de referencia, evitando desplazamientos innecesarios y reduciendo o eliminando estancias hospitalarias.

- Proporcionar una formación continua y de calidad al personal sanitario.

- Aumentar la disponibilidad de recursos de información sanitaria.

- Mejorar la calidad de vida de la población por la posibilidad de recibir asistencia sanitaria personalizada aun en situaciones de aislamiento físico o de difícil acceso.

La telemedicina puede ir desde el intercambio de información sobre el estado de un paciente y los síntomas que presenta vía radio o teléfono para que el médico pueda ayudar al diagnóstico y tratamiento, hasta la asistencia médica o quirúrgica vía videoconferencia (o multiconferencia). Es este último escenario el que le confiere mayor identidad a la telemedicina al permitir introducir otras tecnologías como transmisión de imágenes médicas (radiografías, resonancias magnéticas...) o de signos vitales, y ofrecer, de esta manera, la misma asistencia médica que de forma presencial.

En función de la inmediatez y la forma de comunicación utilizada, se pueden distinguir dos formas de practicar la telemedicina:

- Síncrona o en tiempo real: por ejemplo, cuando se mantiene una sesión de videoconferencia, en la cual el retardo puede ser de fracciones de segundo o de algunos segundos, según el medio de comunicación utilizado.

- Asíncrona: no implica una transmisión en tiempo real; el ejemplo más claro es el envío por correo electrónico de la información en cuestión (estado del paciente, sintomatología, radiografías...) para su estudio por el personal adecuado, quien posteriormente comunicará su diagnóstico por cualquier medio.

\section{LA TELEMEDICINA MILITAR ESPAÑOLA: EVOLUCIÓN HISTÓRICA}

Los orígenes de la telemedicina militar española hay que buscarlos en las primeras consultas por radio de asistencia sanitaria en alta mar. En 1929 se constituyó una comisión para estudiar la creación de un sistema de consultas radiomédicas en los hospitales de la Armada que complementase la presencia de un facultativo en los buques mercantes españoles. Como consecuencia de los trabajos de la comisión, en 1930 quedó establecido el primer 


\section{Hillán García, et al.}

Tabla 1. Tipología de teleconsultas según su prioridad y sistema ${ }^{11}$

\begin{tabular}{|ccc|}
\hline Sistema & Prioridad & Clasificación \\
\hline $\begin{array}{c}\text { VIDEOCONFERENCIA } \\
\text { DATOS }\end{array}$ & URGENTE & CLÍNICA \\
& & Gestión / Técnica / Docente \\
PADIO-TELEFóNICA & PROGRAMADACA & Cestión / Técnica / Docente \\
& URGENTE & CLÍNICA \\
& PROGRAMADA & Gestión / Técnica / Docente \\
CLÍNICA \\
CORREO ELECTRÓNICO & NO en tiempo real & Gestión / Técnica / Docente \\
\end{tabular}

centro español de consultas radiomédicas ${ }^{15}$, con una estación en cada departamento naval, enlazada con el hospital de marina correspondiente, donde las peticiones de ayuda eran atendidas, las 24 horas del día, por el médico de guardia del establecimiento.

En 1968 los servicios de atención radiomédica que venían funcionando en los distintos hospitales navales españoles fueron centralizados en la Policlínica Naval "Ntra. Sra. del Carmen" de Madrid, que acababa de ser inaugurada. Desde allí, y durante diez años, se atendieron las consultas de todos los buques de las flotas mercante y pesquera que llegaban a través de las estaciones de Aranjuez Radio y Pozuelo del Rey Radio ${ }^{15-16}$. No obstante, cuando las llamadas eran por onda media o VHF, éstas eran recogidas por parte de la Red de Estaciones Radio-Costeras Litorales, desde donde se pasaban, en el mejor de los casos, a hospitales públicos o privados ${ }^{16}$. El primer telegrama que se conserva de este servicio corresponde a una consulta solicitada desde el "San Marcial", cursado el 14 de abril de 1969, mientras que el último registrado lleva fecha de 6 de septiembre de $1981^{15}$.

En 1979 el Centro Radio-Médico Español (CRME) dependiente del Instituto Social de la Marina (ISM), inicia su actividad y toma poco a poco el testigo de los servicios de atención radiomédica para ofrecer asistencia médica gratuita a todos los trabajadores del mar con independencia de su nacionalidad y área de navegación. Se comienza utilizando radiotelegrafía, más tarde radiotelefonía y a partir del 2000 la mayor parte de las comunicaciones se realizan vía satélite.

Un hecho que marcaría la historia de la telemedicina en España, aparece referenciado en la Revista Medicina Militar ${ }^{(1)}$ en 1985, donde se hace eco del primer electrocardiograma realizado por teléfono durante el LV crucero de instrucción del Buque-Escuela "Juan Sebastián El Cano" cuando navegaba rumbo Buenos Aires. El propio Teniente Médico Jefe de Sanidad del Buque por aquellos años, Francisco de Asís Fernández Riestra, narra con detalle las dos pruebas realizadas en alta mar con un transmisor RE-130 de antena de látigo y un aparato de telecardio CMT 248M ${ }^{12}$.

Esta experiencia adquirió gran relevancia dentro de la historia de la telemedicina española, al suponer el primer barco español capaz de realizar un electrocardiograma y transmitir con éxito el trazado EKG del paciente desde alta mar y a gran distancia de la base. Desde ese momento se abre la posibilidad de dotar a los buques de recursos para diagnosticar enfermedades cardiacas y recibir atenciones médicas relacionadas.

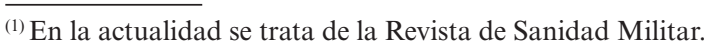

No obstante, hubo que esperar 11 años para que en 1996 se inaugurara la Unidad de Telemedicina en el Hospital Central de la Defensa "Gómez Ulla" (2) que es considerado el primer servicio de telemedicina que se habilitó en España. Su misión era la provisión de servicios sanitarios a las unidades del ejército español desplegados y a los buques de la Armada. Pero, posteriormente, sus servicios se extenderían a otros ámbitos fuera de las Fuerzas Armadas españolas (FAS). Con esta unidad se pone las bases del actual Sistema de Telemedicina de las Fuerzas Armadas españolas y se inicia una nueva etapa de desarrollo y mejora cualitativa en la telemedicina miliar española, que no ha cesado desde entonces.

El primer servicio de telemedicina que se prestó en esta Unidad fue en 1996 entre el Escalón Médico Avanzado del Ejército de Tierra EMET desplegado en Mostar (Bosnia-Herzegovina) en operaciones de mantenimiento de la paz, y el entonces Hospital Militar Central Universitario "Gómez Ulla". Se estableció una sesión de videoconferencia vía satélite Inmarsat con terminales Tandberg Vision conectados a través de la sección de comunicaciones de la empresa Tecnobit en colaboración con Hispasat. Esta sección de comunicaciones más tarde se escindiría dando lugar a la empresa Comitas y su Red TM-64, a la que dedicaremos unas líneas más adelante. Los enlaces se realizaron por medio de una línea RDSI de 64 Kilobytes por segundo (Kbps), posibilitando la transmisión de placas radiológicas de fracturas óseas de una calidad óptima para esos fines.

Este hecho marcaría un antes y un después en la historia de la telemedicina militar española; el éxito y las lecciones aprendidas de la sesión de videoconferencia en la campaña de BosniaHerzegovina en 1996, encumbran y consolidan el papel fundamental de la telemedicina dentro de la Sanidad Militar y pone los cimientos de una carrera de prospección de la telemedicina que se percibe infinita.

\section{La red sanitaria de las Fuerzas Armadas Españolas a lo largo del tiempo}

A medida que la telemedicina cobraba importancia en la Sanidad Militar, la Red Sanitaria de las FAS ha ido cambiando y adaptándose a las necesidades del momento. Pero, antes de co-

\footnotetext{
(2) Entonces el Hospital Central de la Defensa "Gómez Ulla" se denominaba Hospital Militar Central Universitario "Gómez Ulla".
} 


\section{El Sistema de Telemedicina Militar en España: una aproximación histórica}

nocer su evolución, es necesario entender la estructura y tipo de centros con los que se cuentan en la Sanidad Militar.

En el Ministerio de Defensa existen unas Unidades que pertenecen al Órgano Central del Ministerio, al Ejército de Tierra, a la Armada o al Ejército del Aire, y que forman parte de la Sanidad Militar. Todas ellas tienen la misión de atender la salud de los miembros de las Fuerzas Armadas y constituyen el despliegue sanitario del Ministerio de Defensa.

En general se distinguen dos tipos de centros, en función de su capacidad de asistencia médica y del equipamiento de telemedicina de que disponen:

- Centro de referencia: dispone de una elevada variedad de personal médico, de distintas especialidades, alertados y preparados para prestar sus servicios en cuestión de pocos minutos. Este equipo médico está a disposición de cualquier otro centro que requiera sus servicios. De ello se deduce que el equipamiento técnico de telemedicina de que dispone el Centro de Referencia es mayor y más especializado que el de cualquier centro remoto, pudiendo contar con monitores especiales de presentación de imágenes, sistemas de grabación y almacenamiento de imágenes y datos y una mayor infraestructura de comunicaciones, así como equipos de video Dual y Telestrator (posibilidad de dibujar sobre el video remoto).

- Centro remoto: este centro es el que normalmente requiere de los servicios prestados por el centro de referencia. $\mathrm{Su}$ equipamiento técnico incluye además de los sistemas de videoconferencia, los distintos sensores/transductores que se aplicarán al paciente, y cuya información llegará al centro de referencia para su interpretación y uso por el personal especializado presente en este centro. Centros Remotos son las Unidades embarcadas y desplazadas, es decir, los buques de la Armada, las expediciones, las misiones internacionales, etc.

Aunque la mayor parte de las comunicaciones se establecen entre centro remoto-centro de referencia, desde 2010 se habilitó la posibilidad de que cualquier centro remoto pudiese además establecer comunicación con cualquier otro centro remoto, lo cual, ha supuesto un paso más en la telemedicina de las FAS, al posibilitar el intercambio de información entre toda la infraestructura de la sanidad militar y, con ello, la mejora en la toma de decisiones y el ejercicio de la telemedicina.

Desde los inicios en 1996 hasta 2002 se contaba con la Unidad de Telemedicina en el Hospital Central de la Defensa "Gómez Ulla" como único centro de referencia. Fue en este mismo año, cuando se habilitaron los equipos de telemedicina en Afganistán y en Irak, abriéndose una gran puerta al uso de la telemedicina, y posibilitando la dotación de equipos de telemedicina a otros centros remotos y a otros centros hospitalarios.

Durante aproximadamente 11 años, la Unidad de Telemedicina será dependiente del entonces Servicio de Medicina Logística que se encargaba de dar soporte y proveer de personal médico a las unidades del ejercicio en operaciones. Con el inevitable progreso en la provisión de asistencia sanitaria a distancia, las tareas de la Unidad de Telemedicina van cobrando mayor relevancia a lo largo de los años, y a su labor, van incorporándose otros centros de apoyo a la telemedicina dentro de la Red Hospitalaria Militar, de tal forma, que al término del año 2006, se contaba con 8 centros habilitados con equipos de telemedicina dentro de su Red Hospitalaria. Es en ese punto, cuando la Unidad de Telemedicina del Hospital Central de Defensa "Gómez Ulla" pasa a depender de la Secretaría Técnica para, de esta forma, dar respuesta a las exigencias y necesidades del momento. Y unos años después, en el 2010, la Unidad de Telemedicina, en su ánimo de progreso, se constituirá como Servicio de Telemedicina, tal como se le conoce hoy en día.

Los centros de telemedicina de la Red Hospitalaria con los que se contaban en el 2006 estaban ubicados en: el Hospital Central de la Defensa "Gómez Ulla" en Madrid; en el Hospital General de la Defensa "Orad Cagigas" en Zaragoza; en el Hospital General de la Defensa en San Fernando; el Hospital GeneralBásico de la Defensa en Valencia; en el Hospital General-Básico de la Defensa en El Ferrol; en el Hospital General de la Defensa en Cartagena; en el Hospital Militar de Ceuta, y en el Hospital Militar de Melilla ${ }^{17}$.

En la actualidad, el Centro de Referencia por excelencia sigue siendo el Servicio de Telemedicina del Hospital Central de la Defensa "Gómez Ulla" apoyado por otras unidades ubicadas en el Hospital General de la Defensa de Zaragoza, y el Hospital General Básico de la Defensa de San Fernando. El resto de centros hospitalarios que en su día estuvieron dotados con equipos de telemedicina, han pasado a ser clínicas militares y han ido delegando sus competencias en telemedicina al Hospital Central de la Defensa "Gómez Ulla".

En cuanto a las instalaciones de equipos de telemedicina en centros remotos se han ido incorporando en función de la presencia de las Fuerzas Armadas españolas en zonas remotas y en misiones internacionales de cada momento.

A continuación se nombran los centros remotos agrupados por ejércitos que han ido instalándose a lo largo del tiempo:

\section{En el Ejercicio de Tierra:}

- Base España en Istok (Kosovo), fuera de servicio por la retirada de las tropas española en 2010.

- Base de Qala-e-Naw (Afganistán).

- Base Antártica Gabriel de Castilla.

- Base Miguel de Cervantes en Marjayoun (Líbano).

- Islas Chafarinas, Peñón de Vélez y Peñón de Alhucemas.

- Las Agrupaciones de Sanidad de la Brigada de Sanidad y su Unidad de Apoyo Logístico Sanitario. (Base de Qalae-Naw y COP,s de Ludina y Mucqur en Afganistán).

En la Armada:

- Buques de desembarco Galicia y Castilla.

- Buque de apoyo al combate Patiño.

- Buque-Escuela Juan Sebastián de Elcano.

- Buque de Apoyo al Combate (BAC) Cantabria.

- Buque de Proyección Estratégica Juan Carlos Primero.

- Fragatas F100, Álvaro de Bazán, Méndez Núñez y Blas de Lezo.

- Patrulleros de altura Tarifa, Alborán, Arnomendi y Chilrreu

- Tercio de Armada de Infantería de Marina. 


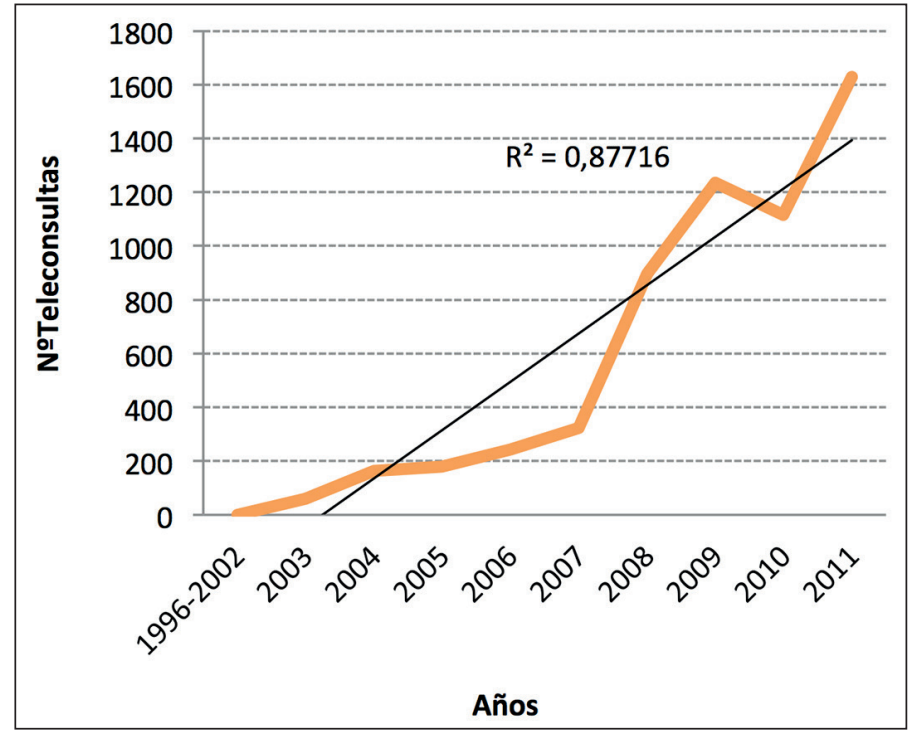

Figura 2. Número de teleconsultas del Servicio de Telemedicina ${ }^{19}$.

En Ejército del Aire:

- Base de Herat en Afganistán.

- Escuadrón de Vigilancia Aérea (EVA) número 5, en la Sierra de Aitana (Alicante).

- EVA número 7, en Soller (Mallorca).

- EVA número 11, en Alcalá de los Gazules (Cádiz).

- EVA número 12, en Espinosa de los Monteros (Burgos).

- EVA número 21, en Pico de las Nieves en San Mateo (Las Palmas)

- Unidades Médicas Aéreas de Apoyo al Despliegue, en la base aérea de Torrejón y en Zaragoza.

- Unidad Médica de Aeroevacuación en la base aérea de Torrejón.

\section{Recursos humanos y actividades del Servicio}

Desde que en 1996 se inaugurara la Unidad de Telemedicina al mando del entonces Capitán Médico Alberto Hernández Abadía de Barbará, se ha asistido a un incremento significativo de los indicadores de desempeño de este servicio. No sólo, se ha aumentado el número de teleconsultas que se realizan al año, sino que, además, se han aumentado las competencias, y se han diversificado los servicios y actividades que se prestan.

A lo largo de estos 16 años de actividad se ha pasado de ofrecer un servicio prioritariamente logístico-operativo a un servicio integral de telemedicina en el que se lleva a cabo funciones de tipo asistencial, logístico-operativo, de docencia, de investigación, de consultoría, y de apoyo a la medicina pericial. A día de hoy, los servicios y actividades que se realizan son:

- Teleconsultas médicas: que ofrecen asesoramiento y/o asistencia médica en sesiones urgentes o programadas.

- Asistencias técnicas sobre logística y equipos de telemedicina.
- Sesiones clínicas inter-hospitalarias: que se inician a comienzos del 2004 para compartir conocimientos sobre temas específicos en tiempo real ${ }^{18}$.

- Formación continua en Telemedicina: se capacita al personal sanitario que trabajará en centros remotos, tanto en Territorio Nacional como en buques, expediciones y Zonas de Operaciones.

- Investigación: se analizan y se testean nuevos procedimientos, y nuevos equipos y sistemas, con el objetivo de impulsar la innovación y de incorporar mejoras.

- Consultoría: se colabora en grupos de trabajo nacionales e internacionales sobre normalización y transmisión de información sanitaria.

- Pericial y otros: Soporte a otras unidades, como por ejemplo, ofrecer los equipos de telemedicina para la comparecencia en juicios por videoconferencia de médicos especialistas.

Si analizamos la evolución anual del porcentaje de teleconsultas que se han atendido a lo largo del tiempo, se observa un crecimiento significativo a partir del año 2007, alcanzando máximos históricos en el 2009, y con fuerte tendencia al alza para los años futuros, según muestra la línea de tendencia de regresión lineal y el valor cercano a 1 del indicador estadístico $\mathrm{R}^{2}$.

Este aumento de actividad coincide con una mejora de las capacidades y de la proyección del Servicio de Telemedicina, sobre todo a partir del 2007. Además de las continuas inversiones en la mejora de los equipos y las comunicaciones, los servicios de telemedicina comienzan a tener mayor transcendencia dentro del marco de trabajo del Ministerio de Defensa para mejorar la política exterior de España. Así por ejemplo, en 2008, se incorporan otros dos equipos de telemedicina (centros remotos) en el Hospital Militar de Nouakchott, en Mauritania y en el Hospital de Abdulah Nakas, en Sarajevo ${ }^{20}$.

Asimismo hay que tener en cuenta que la actividad del Sistema de Telemedicina, no se ciñe al entorno militar, sino que se extiende también al ámbito civil. En 2007 el Ministerio de Defensa y el Instituto Social de la Marina firman un convenio de colaboración para la asistencia telemática por parte del Hospital Central de la Defensa "Gómez Ulla" a la flota de buques sanitarios y de salvamento marítimo Esperanza del Mar y Juan de la Cosa, y al Centro Radio Médico Español ${ }^{3}$. También se colabora con Organizaciones No-Gubernamentales (ONGs) tales como la Fundación Chinguetti ${ }^{21}$ para apoyar al personal destacado en Mauritania.

Por el servicio de telemedicina han pasado buenos profesionales médicos, ingenieros, técnicos, enfermeros, y hasta documentalistas. No obstante, todos aquellos que conocen el Servicio no pueden por menos que asociarlo con aquella persona que ha sido alma mater y catalizador de la prosperidad del Servicio de Telemedicina. Hablamos del entonces Capitán, y hoy Teniente Coronel Médico Alberto Hernández Abadía de Barbará quien al frente de la Unidad desde 1996, consigue impulsar su desarrollo y hacer de la telemedicina militar española un referente dentro y fuera de nuestras fronteras. A sus espaldas quedan más de 15 años de empeño, innovación y mejora de la telemedicina militar que no ha dejado indiferente a nadie. A partir del 2002 se uniría a esta gran aventura el entonces Teniente Ricardo Melgarejo Cristóbal cuya labor ha sido de vital 


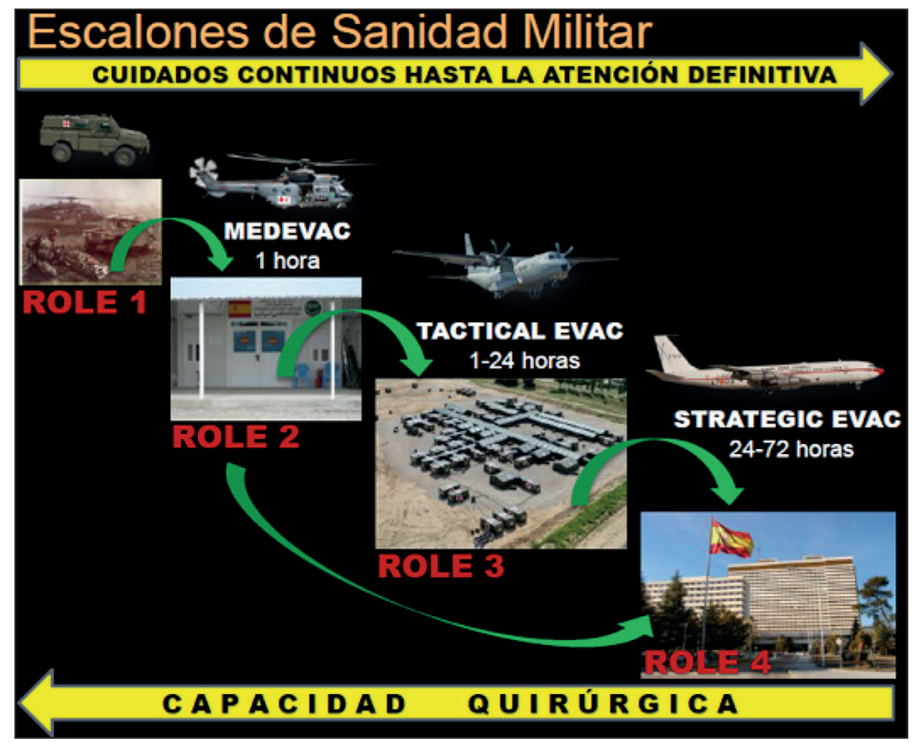

Figura 3. Escalones de Sanidad Militar ${ }^{23}$.

importancia para la buena marcha del Servicio durante los 10 años que prestaría sus servicios, primero como teniente y, más tarde como Capitán.

Desde septiembre del 2012 el Comandante Médico Antonio del Real Colomo toma el testigo de la dirección del Servicio para retomar la labor desempeñada por su antecesor. Se abre, por tanto, una nueva etapa de buenos propósitos, ilusiones y grandes expectativas que, seguramente, merecerá otro capítulo en la historia de la telemedicina militar española.

\section{Funcionamiento}

La provisión de telemedicina en las FAS está estrechamente relacionada con el modelo operativo que se sigue en la asistencia sanitaria militar, el cual se ha mantenido prácticamente inalterable a lo largo del tiempo.

Cuando se realiza un despliegue en cualquier misión internacional, la Sanidad Militar define una cadena de asistencia y evacuación, con distintas situaciones físicas y capacidades médicas. Esta cadena está compuesta por 4 escalones, denominados Role 1, Role 2, Role 3, y Role 4. Los 3 primeros Roles están en la zona de despliegue o en sus cercanías, mientras que el Role 4 se sitúa en territorio nacional ${ }^{22}$. Forman parte de este último, los centros de la Red Hospitalaria y, muy especialmente, el Servicio de Telemedicina o Centro de Referencia del HCD "Gómez Ulla".

Las funciones de los diferentes Roles son las siguientes:

- Role 1: físicamente situado en la zona de despliegue, sus funciones son proporcionar atención médica en la vanguardia de la acción, atendiendo las urgencias in situ, prestando primeros auxilios, reanimación, estabilización de funciones vitales, selección y clasificación (triaje o tria$\left.\mathrm{ge}^{(3)}\right)$. Los pacientes se tratan al momento, o bien se pre-

(3) Habitualmente, en situaciones de emergencia o desastre, se le llama "triaje o triage" a la clasificación de heridos según su estado y gravedad, para para su desplazamiento en función de la gravedad de su estado clínico.

- Role 2: situado en las inmediaciones de la zona de vanguardia, está formado por la estructura capaz de recibir las bajas provenientes del Role 1, y proporcionar estabilización, selección y clasificación de dichas bajas, que serán tratadas, incluso con cirugía básica, para ser devueltos a su condición operativa o bien para ser evacuados. En el segundo escalón ó Role 2 se dispone de los siguientes tipos de Unidades:

EMAT: Escalón Médico Avanzado del Ejército de Tierra.

UASAN: Unidad de Asistencia Sanitaria del Ejército de Tierra.

USANEM: Unidades de Sanidad Embarcadas de la Armada.

UMAAD: Unidad Médica de Apoyo Aéreo al Despliegue del Ejército del Aire.

UMAER: Unidad Médica de Aeroevacuación del Ejército del Aire.

- Role 3: Este escalón se materializa habitualmente en un hospital de campaña, que puede estar incluso embarcado.

- Role 4: corresponde a los hospitales existentes en la infraestructura nacional de Sanidad Militar, y realiza el tratamiento definitivo a los pacientes.

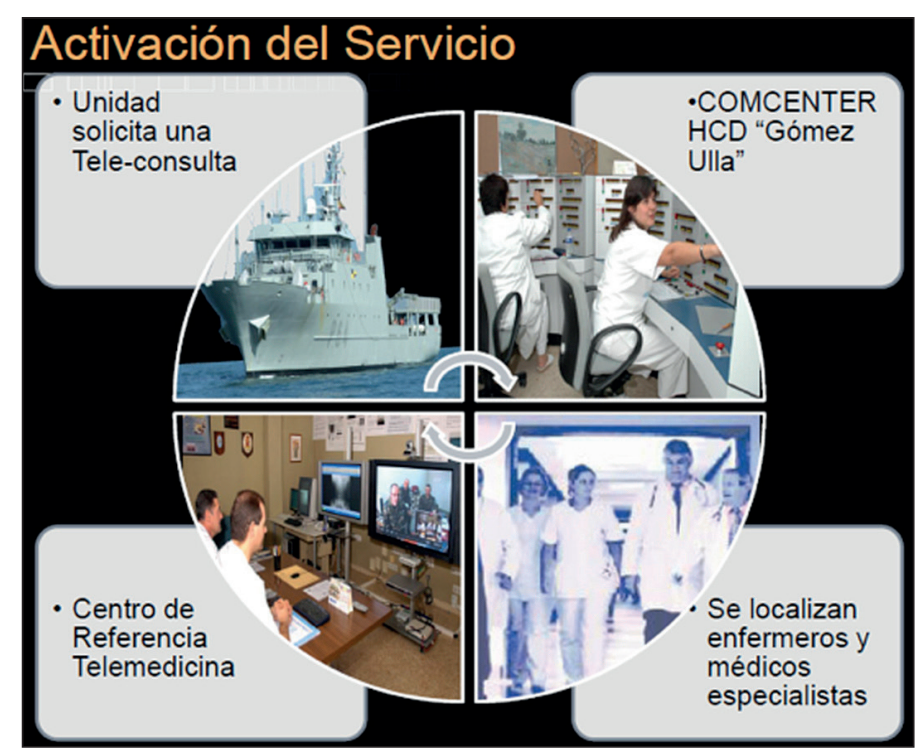

Figura 4. Activación del Servicio de Telemedicina ${ }^{23}$.

Cada escalón debe tener obligatoriamente las capacidades definidas para su nivel y además las definidas para los niveles inferiores. Además, los requisitos de movilidad son, evidentemente, diferentes para cada uno. Así, por ejemplo, en los hospitales de territorio nacional se puede disponer de equipos de videoconferencia instalados que no necesitan ser desplazados por estar situados en las salas habilitadas para realizar las teleconsultas.

establecer prioridades y su posterior tratamiento o evacuación de manera separada 


\section{Hillán García, et al.}

En las instalaciones de Role 2 ó 3, por el contrario, puede ser necesario tener equipos que permitan cierta flexibilidad para desplazarlos en ciertos casos. De igual manera, también son distintos los requisitos exigidos al equipamiento de comunicaciones: en los centros nacionales, se requiere mucha versatilidad (enlaces por distintos medios, cableados/inalámbricos, redundancia, elevado ancho de banda que incluso permita mantener varias videoconferencias simultáneas con diferentes centros), mientras que en las unidades desplazadas no se suele disponer de mucho ancho de banda ni tampoco de la posibilidad de tener enlaces duplicados de seguridad, aunque sí es deseable poder utilizar distintos medios de comunicación para prever eventualidades. También son distintos los requisitos referidos al equipamiento en general (sensores/transductores y equipamiento informático "ruguerizado" o militarizado para Roles 1, 2 y posiblemente 3; más completos en Role 4 que en Role 1), así como los de acceso a la información, los de seguridad, etc.

La labor del cuarto escalón o Role 4, donde se encuentra el Centro de Referencia del HCD "Gómez Ulla", es clave por cuanto facilita que puedan ser interconectados la totalidad de instalaciones de tratamiento médico para apoyar al despliegue y para la adquisición de información médica sobre el terreno.

La activación del Servicio de Telemedicina proviene de una llamada por parte de la Unidad que tiene una necesidad médica. Esta llamada es recibida en el Comcenter del HCD "Gómez Ulla" que se encargan de localizar a los enfermeros y médicos especialistas, y que posteriormente se dirigirán al Centro de Referencia del Hospital para realizar la videoconferencia. Se trata del único servicio en España que presta asistencia sanitaria a distancia las 24 horas del día los 365 días del año. Cabe mencionar, como elemento innovador de este Servicio, que en el caso de que el personal adscrito al servicio no se encuentre en el Hospital, el jefe médico del Servicio de Telemedicina podrá realizar asistencia técnica y médica de la videoconferencia a través de lo que se ha denominado "Gran Hermano"22, un sistema de telecomunicaciones que funciona con tecnología móvil 3G.

Con este Sistema de Telemedicina lo que se pretende en última instancia es: conseguir un diagnóstico preciso de la dolencia del paciente; prescribir el tratamiento adecuado para la misma; estabilizar al paciente en caso que fuese necesario; decidir la necesidad o no de traslado; y supervisar su evolución en caso de no ser trasladado. Es de vital importancia la decisión de traslado de un paciente, ya que de no valorarse adecuadamente esta necesitad se podría gestionar una evacuación innecesaria desde las zonas de despliegue con el consiguiente riesgo de agravamiento de las lesiones del paciente, la puesta en peligro de su seguridad y la del personal que lo acompaña, así como la ineficiencia en la provisión de recursos que pudiera afectar al equilibrio de los equipos médicos.

\section{Tecnología y equipos de telemedicina}

La evolución en los sistemas y equipos de telemedicina con los que se ha contado en la Sanidad Militar española ha ido al compás del desarrollo tecnológico y de las comunicaciones.

Como se veía en los inicios de la telemedicina y queda referenciado ${ }^{6,24}$, los enlaces por radio eran la forma más común de comunicarse entre dos puntos para ofrecer asistencia médica hasta que a partir del año 1996 se empezaron a instalar nuevos sistema de comunicación bidireccional que permitían el intercambio de audio, video y datos de forma simultánea, interactiva y en tiempo real, como son los sistemas de videoconferencia.

Este tipo de tecnologías necesita de una señal digitalizada que se transmite vía terrestre o por satélite a grandes velocidades. Dentro de la telemedicina militar española, cuando las comunicaciones se establecen entre la Red Hospitalaria las conexiones se establecen vía terrestre, y cuando se establece comunicaciones con los centros remotos, las conexiones son, en su mayor parte, vía satélite.

Aunque desde 1996 hasta 2002 las teleconsultas que se realizaban eran sobre todo vía terrestre, ya se contaba con terminales Tandberg Vision que permitían una conexión vía satélite Inmarsat a través de una simulación de línea RDSI (Red Digital de Servicios Integrados) de 64 Kbps.

Un avance importante en la telemedicina militar española fue cuando en 2007 se dotó a los Buques de la Armada de terminales tipo $\mathrm{F} 77^{13}$, al aumentar el doble la capacidad de ancho de banda, es decir, hasta los $128 \mathrm{Kbps}$, y por tanto, ampliando las prestaciones de los equipos y la velocidad de las comunicaciones. Anteriormente los buques contaban con un solo terminal de tipo Saturno Bravo de $64 \mathrm{Kbps}$, lo que suponía, la mitad del ancho de banda actual y por ende, la mitad de prestaciones y de velocidad.

En la actualidad, tanto para el Ejército de Tierra como para el Ejército del Aire se utilizan terminales tipo NERA M4 que permiten una conexión de $64 \mathrm{Kbps}$ de ancho de banda cada uno o de $128 \mathrm{Kbps}$ con 2 terminales satélite. Además, se cuenta también con terminales tipo BGAN (la nueva generación de Inmarsat) que permiten una conexión de $256 \mathrm{Kbps}$. Para la Armada, los buques cuentan con los ya nombrados terminales F77 de $128 \mathrm{Kbps}$.

Estos equipos permiten realizar:

- Videoconferencia en tiempo real (VTC).

- Ultrasonografía en tiempo real: ecografía abdominal, ecocardiografía, ecografía de partes blandas, etc.

- Telemetría: frecuencia cardiaca, tensión arterial no invasiva, saturación periférica de oxígeno y temperatura.

- Electrocardiografía de 12 derivaciones en tiempo real.

- Transmisión de otros datos: analíticas, historias electrónicas, etc.

- Transmisión de pruebas de imagen: tanto preparaciones microscópicas de anatomía patológica, imágenes de alta calidad dermatológicas como radiografías, tomografía axial computadorizada, resonancia magnética nuclear y el resto de técnicas de diagnóstico por la imagen que se imprimen sobre soportes físicos como el acetato.

\section{Conexiones vía satélite}

La tecnología de comunicación por satélite para la interconexión entre puntos, fue sin duda uno de los grandes saltos en la evolución de la telemedicina, y lo que le confiere mayor identidad a esta disciplina.

En la telemedicina militar española se cuenta en estos momentos con dos redes satelitales distintas cuyo uso ha ido evo- 


\section{El Sistema de Telemedicina Militar en España: una aproximación histórica}

lucionando a lo largo del tiempo y ha estado marcada por las prestaciones y necesidades de cobertura de comunicación: la Red Inmarsat y la Red SECOMSAT.

La Red Inmarsat es una red de comunicaciones de uso general internacional en la que Defensa tiene contratado unos canales de comunicación. Estos canales permiten unas velocidades de transmisión de hasta 2 Megabytes por segundo (Mbps) y dan cobertura a más del 90\% de la superficie terrestre. Esto permite dar cobertura zonas aisladas y difícil acceso como es la Antártica. La Red Inmarsat fue la primera red satelital con la que se contó en el Servicio de Telemedicina para realizar las primeras sesiones por videoconferencia. Pero los problemas de saturación de la red en determinadas franjas horarias, así como el alto coste de su uso (aprox. \$6/ min), hicieron plantearse otras alternativas de conexión vía satélite, y a partir del 2007 se comienza a utilizar la Red SECOMSAT para todas aquellas conexiones dentro de su alcance de cobertura.

La Red SECOMSAT es una red de comunicaciones española en la cual Defensa tiene unos canales de comunicación propios y protegidos, de tal manera que no se ven afectados en la velocidad de transmisión por otros usuarios de la red como sucede con la Red Inmarsat, y además se reducen significativamente los costes. El único inconveniente en el uso de SECOMSAT es que los anchos de banda disponibles para la transmisión se han de compartir con el resto de servicios militares, y que su cobertura no alcanza todos los puntos geográficos del planeta.

\section{La Red de Telemedicina TM-64}

Con la campaña de Bosnia-Herzegovina en 1996 se pone las bases para el desarrollo de una red temática sanitaria capaz de ofrecer un canal de comunicación independiente con fines exclusivos de telemedicina: La Red de Telemedicina TM-64.

La Red TM-64 de la empresa Comitas se caracteriza porque opera sobre una red IP dedicada, y por tanto está aislada de Internet. Esta red permite la transmisión de datos e imágenes de alta resolución con el objetivo de ofrecer asistencia médica en tiempo real a pacientes ubicados en cualquier lugar del mundo. Además es también utilizada para realizar sesiones clínicas, conferencias y cursos de formación.
La comunicación se puede realizar tanto por vía satélite como por circuitos terrestres dedicados con un ancho de banda mínimo de $64 \mathrm{Kbps}$ y de hasta $2 \mathrm{Mbps}$. Todos los circuitos están protegidos por equipos de encriptación ${ }^{25}$ para garantizar una total confidencialidad.

Aunque la actividad de la Sanidad Militar fue la causa de la creación y el desarrollo de esta red temática sanitaria, hoy en día, se encuentran integrados, además de los centros y nudos operados por la Sanidad Militar, otros centros sanitarios públicos y privados distribuidos por toda la geografía nacional así como buques e instalaciones sanitarias fuera del mismo.

\section{Perspectivas de futuro}

Los esfuerzos en telemedicina militar van encaminados a conseguir que todos los niveles de atención sanitaria en las Fuerzas Armadas se intercomuniquen a través del Sistema de Telemedicina según sus despliegues, roles y necesidades ${ }^{26}$. A su vez, y como consecuencia de lo anterior, la formación continua en telemedicina, se debe extender a todo el personal de Sanidad Militar incluso incorporar dentro de los planes de carrera universitarios del área de sanidad.

La apuesta de futuro del Servicio de Telemedicina gira en torno a una filosofía de mejora continua basada en la inversión en $\mathrm{I}+\mathrm{D}+\mathrm{i}$, fruto de lo cual, están en marcha varios proyectos en las siguientes líneas de investigación:

- Telemedicina de combate (eSafeTag): con el objetivo de ampliar la Telemedicina hasta el punto donde se produzca la baja (Primeros Escalones Sanitarios). Dentro de esta área se experimenta con los nuevos uniformes de los combatientes del futuro (COMFUT) y las Tarjetas digitales con datos clínicos individuales (Personal Information Carrier ó MiTAG).

- Estandarización de protocolos, procedimientos y formatos: en el contexto nacional a través de la colaboración con otros hospitales e instituciones, y en el contexto internacional a través de la participación activa en el Panel de Expertos MEDCIS (Medical Information Management System) y TMED Expert Team del COMEDS de la OTAN.

Tabla 2. Hitos en la historia de la telemedicina militar española.

1985 Transmisión de un electrocardiograma por radio ${ }^{12}$.

1996 Conexión por videoconferencia "Tandberg Vision" por satélite Inmarsat desde la Unidad de Telemedicina del HMCU "Gómez Ulla" al Escalón Médico Avanzado del Ejército de Tierra EMAT desplegado en Mostar (Bosnia i Herzegovina).

2002 Se adquieren los equipos. Inicialmente el sistema de videoconferencia es por RDSI hasta 384 Kbps ${ }^{4}$. Los centros terrestres (Hospitales Militares) se conectan a $512 \mathrm{Kbps}$ por RDSI. Y los centros remotos vía Inmarsat a 64 Kbps o $128 \mathrm{Kbps}$.

2006 Expedición cívico-militar al Gasherbrum II, en Pakistán.

2007 Se comienza a utilizar la Red Satelital de SECOMSAT en el Servicio de Telemedicina. Las fuerzas armadas disponen de participación en un satélite y lo gestionan directamente utilizando la tecnología DVB-RCS.

2007 Dotación de terminales F77 a los Buques de la Armada ${ }^{13}$.

2009 Simulacro de teleconsulta y envío en tiempo real una imagen de ecografía cardiaca desde los montes Altai en Mongolia a la Unidad Central de Telemedicina del Hospital Central de la Defensa Gómez Ulla en Madrid ${ }^{14}$. 


\section{Hillán García, et al.}

- Plataformas informáticas de telemedicina (T4MOD): desarrollar un sistema de comunicación satélite de nueva generación de 2 Mbps simétricos que permita la realización de teleconsultas en tiempo real entre las MTF (Medical Treatment Facilities) y los hospitales militares de referencia de cuatro países (Alemania, Francia, Italia y España), en zonas de operaciones. Asimismo con esta plataforma se persigue potenciar la teleasistencia quirúrgica en tiempo real y en alta definición, y mejorar la teleecografía

- Telemedicina móvil y telemedicina 3G-4G: para dotar a las ambulancias tácticas de las FAS de equipos de telemedicina de última generación y para posibilitar la realización de teleconsultas en tiempo real con terminales móviles de cobertura 3G-4G. Esto permitirá tener un sistema de alertas de todas las especialidades y, por tanto, una alta accesibilidad y disponibilidad de los servicios de telemedicina.

- Gestión y redes de información sanitaria: la Red de Telemedicina de Sanidad Militar será una parte fundamental del Sistema de Información Sanitaria de la Defensa (BALMIS). Este Sistema permitirá la interoperabilidad con el Sistema Logístico Operativo de Sanidad (SILOPFASSAN) y con el Sistema de Información Sanitaria de la OTAN (MEDICS). Por otra parte, está en marcha un proyecto multicéntrico de recogida centralizada de datos médicos para la realización de estudios sobre alarmas inteligentes.

- Capacitación a distancia con nuevos dispositivos: para mejorar la práctica de la telemedicina se realizan diversos estudios de capacitación a distancia con nueva instrumentación para conocer las ventajas que puede aportar el uso de estas nuevas herramientas, y proceder a su incorporación si se considera necesario.

\section{CONCLUSIONES}

Nadie parece dudar del potencial de la telemedicina, y de las oportunidades que ésta puede ofrecer en la provisión de la cobertura sanitaria, la equidad en el acceso, la calidad en los servicios o la eficiencia en la utilización de los recursos médicos ${ }^{5,10,27-29}$. A la luz de este potencial, la Organización Nacional de la Salud (OMS) abrió el Observatorio Global para la eHealth (GOe) con el objetivo de analizar los beneficios que las TIC pueden traer a la asistencia médica y al bienestar del paciente?

Sin embargo, aún quedan retos y barreras que superar entorno al uso de la telemedicina, entre los que se suelen citar: la resistencia a la adopción de modelos de servicios innovadores; la carencia de formación en aplicaciones de telemedicina; la falta de estudios que demuestren los beneficios económicos y la rentabilidad de la telemedicina, y la ausencia de un marco jurídico internacional que permita a los profesionales de la salud prestar servicios en diferentes jurisdicciones y países. Y a pesar de estas limitaciones, la telemedicina ha experimentado, en un corto periodo de tiempo, un rápido crecimiento acompañado por los avances en las TIC e intensificado por unas necesidades y demanda del paciente cada vez mayores a los que dar respuesta.
Un buen ejemplo del importante papel que ejerce la telemedicina en la sanidad lo encontramos en las Fuerzas Armadas Españolas; el gran desarrollo que ha experimentado esta disciplina en el mundo militar en poco años, nos da una idea de las enormes ventajas que puede incorporar la telemedicina a la sanidad, y el prometedor futuro que le queda por delante. Las comunicaciones vía satélite con centros remotos de difícil acceso, supuso un gran salto en la Sanidad Militar, y posicionó a su Sistema de Telemedicina como el mejor catalizador en la provisión de una atención sanitaria sostenible y de calidad en cualquier momento y en cualquier parte del planeta.

En la actualidad, se están realizando grandes inversiones en telemedicina, como demuestra su creciente importancia económica $^{2}$, dentro y fuera del ámbito militar. Sin embargo hay que tener en cuenta que el grado de desarrollo, y aplicación de la telemedicina difiere en función de las necesidades y funcionamiento de quién la provee y utiliza, así por ejemplo, en España, las Fuerzas Armadas españolas se sitúan al frente de esta práctica con su Sistema de Telemedicina que da respuesta y cubre las exigencias sanitarias en el ejercicio diario de sus actividades. No en vano, este Sistema de Telemedicina es considerado uno de los pilares en los que se sustenta la Sanidad Militar, y un referente internacional en telemedicina miliar ${ }^{22}$.

Por tanto, no parece difícil imaginar hacia dónde se fijarán y orientarán las nuevas agendas políticas, cuando ante todas las miradas, la telemedicina se presenta como una solución atractiva y factible para la sanidad de todas las naciones. Son pocas las cuestiones en las que existe un acuerdo unánime e internacional, máxime en las condiciones adversas en las que se encuentra la economía mundial, es por esto, que cabe pensar que asistiremos a un desarrollo de la telemedicina aún más impactante que el acontecido hasta el momento, sobre todo si como es de esperar, la telemedicina adopta y aprovecha los continuos avances en las tecnologías de la información y las comunicaciones.

\section{BIBLIOGRAFÍA}

1. The future of medicine Squeezing out the doctor. The Economist. Jun $2^{\text {nd }}-8^{\text {th }}$ 2012. Print Edition. Available from: http://www.economist.com/ node/21556227.

2. Research B. Global Markets for Telemedicine Technologies: BCC Research2012 Contract No.: HLC014E.

3. Martínez-Ramos C. Telemedicina en España. II. Comunidades Autónomas. Sanidad Militar, Marítima y Penitenciaria. Proyectos Humanitarios. Reduca, Recursos Educativos. 2009;1(1):182-202.

4. De Toledo Heras P. Propuesta de un modelo de sistema de telemedicina para la atención sanitaria domiciliaria. Madrid: Universidad Politécnica de Madrid, 2003.

5. Cáceres-Méndez E, Castro-Díaz S, Gómez-Restrepo C, Puyana J. Telemedicina : historia, aplicaciones y nuevas herramientas en el aprendizaje. Universitas Médica. 2011;1(52):11-35.

6. Avanzini Blanco E. Tecnologías para una asistencia sanitaria global: La telemedicina. Documentos de Seguridad y Defensa: tecnologías del espacio aplicadas a la industria y servicios de la defensa. 2011;41:63-85

7. Telemedicine: opportunities and developments in Member States: World Health Organization (WHO), 2010.

8. Ricur G. Telemedicina: generalidades y áreas de aplicación clínicas. In: Caranicero J, Fernández A, editors. Manual de salud electrónica: para directivos de servicios y sistemas de salud. Madrid: Sociedad Española de Informática de la Salud (SEIS); Comisión Económica para América Latina y el Caribe (CEPAL); 2012. p. 171-93. 


\section{El Sistema de Telemedicina Militar en España: una aproximación histórica}

9. What is Telemedicine?, 2012. [cited 2012 2013-02-13]; Available from: http:// www.americantelemed.org/learn/what-is-telemedicine.

10. Plan de telemedicina del INSALUD. Madrid: Dirección General de Organización y Planificación Sanitaría de INSALUD. Subdirección General de Sistemas y Teconologías de la Información del Ministerio de Sanidad y Consumo, 2000.

11. Hernández Abadía de Barbará A. Telemedicina en la Sanidad Militar: experiencias y planes de actuación. Presente y futuro de la Telemedicina en España; 200920 Noviembre; Madrid: Intereconomía conferencias.

12. De Asis Fernández Riestra F. Transmisión de un electrocardiograma por radio. Medicina Militar, Revista de las Fuerzas Armadas de España. 1985;41(1):115-6.

13. Hernández Abadía de Barbará A, Trigueros Martín JL. Sistemas de telemedicina e información sanitaria de las Fuerzas Armadas. In: Carrión Pérez PA, Ródenas Gracía J, Rieta Ibáñez JJ, Sánchez Meléndez C, editors. Telemedicina Ingeniería biomédica. Cuenca: Ediciones de la Universidad de Castilla-La Mancha; 2009. p. 151-61.

14. ExpediciónALTAI Una travesía cívico-militar al macizo de Mongolia pone a prueba los más avanzados sistemas de comunicaciones y telemedicina. Revista Española de Defensa. 2009 Abril:46-7.

15. Gracia Rivas M. La sanidad naval española: historia y evolución. [Barcelona]: Bazán; 1995.

16. Bartolomé Martín JA, Córdoba Paje A. Asistencia médica por radio. I Jornadas cívico-militares de Sanidad organizadas por el Ministerio de Defensa y Ministerio de Sanidad y Consumo; 1986 21-23 Noviembre; Madrid: Centro de Publicaciones, Documentación y Biblioteca del Ministerio de Sanidad y Consumo.

17. Betegón Sanz A. Telemedicina en las Fuerzas Armadas (FAS). Congreso Sostenibilidad; 201217 Mayo; Zaragoza: Asociación de Ingeniéros de Telecomunicación de Aragón (AITAR).

18. Hernández Abadía de Barbará A, Hernández Navarro M. La Telemedicina en la Sanidad Militar Española. In: Zamorano JL, Gil-Loyzaga P, Miravet D, editors. Telemedicina: análisis de la situación actual y perspectivas de futuro. Alcobendas (Madrid): Fundación Vodafone, 2004. p. 233-9.
19. Memoria de actividad del Servicio de Telemedicina (1996-2011). Madrid: Servicio de Telemedicina del Hospital Central de la Defensa "Gómez Ulla", 2012.

20. Senovilla M. Diagnósticos a distancia: la telemedicina permite hoy realizar consultas desde cualquier punto del planeta, y en tiempo real, con los hospitales militares de España. Revista española de defensa. 2008;241:50-3.

21. Hernández Abadía de Barbará A, Trigueros Martín JL. La telemedicina militar española. Puesta al día en urgencias, emergencias y catástrofes. Unión de Sociedades de Emergencias. 2008;8(1):39-42.

22. Hernández Abadía de Barbará A. The Spanish Ministry of Defence (MOD) Telemedicine System. In: Graschew G, Roelofs TA, editors. Advances in Telemedicine: Technologies, Enabling Factors and Scenarios. Croacia: InTech, 2011. p. 379-96.

23. Moreno Caravaca AJ. Archívo fotográfico del Servicio del Telemedicna del Hospital Central de la Defensa "Gómez Ulla". Madrid, 2012.

24. Guiote Linares M, Balanya Vidal J, Hernández Abadía de Barbará A, Trapero M. Uniform Sierra: Protocolo de transmisiones ante una urgencia sanitaria en campaña. Medicina Militar Revista de sanidad de las Fuerzas Armadas. 1994;50(5):547-51.

25. Comitas. Telemedicina ¿Qué es la TM64? 2012 [2012-08-22].

26. Hernández Abadía de Barbará A. Telemedicina en las Fuerzas Armadas Españolas. In: Maimir Jané F, editor. Manual de Asistencia al paciente crítico de las fuerzas armadas. Madrid: Ministerio de Defensa, Inspección General de Sanidad de la Defensa, 2004. p. 258-63.

27. Plan de acción sobre la salud electrónica 2012-2020: atención sanitaria innovadora para el siglo XXI. In: Comunicación de la Comisión al Parlamento Europeo, al Comité Económico y Social Europeo, al Comité de las Regiones. Bélgica: Bruselas, 2012

28. Nouhi M, Fayaz-Bakhsh A, Mohamadi E. Telemedicine and Its Potential Impacts on Reducing Inequalities in Access to Health Manpower. Telemed e-Health. 2012;18(8).

29. Monteagudo JL, Serrano L, Hernández Salvador C. La telemedicina: ¿ciencia o ficción? Anales del Sistema Sanitario de Navarra. 2005;28(3):309-23. 